\title{
International and Turkish information technology standardization *
}

\author{
Behçet Sarikaya ${ }^{a}$ and Rasim Yilmaz ${ }^{b}$ \\ ${ }^{a}$ Bilkent University, Dept. of Computer Engineering, and Information Sciences Bilkent, Ankara 06533, Turkey \\ ${ }^{b}$ Computer Center Director, Turkish Standards Institute, Necatibey Cad. No. 112, Bakanliklar, Ankara 06100, Turkey
}

\begin{abstract}
Sarikaya, B. and R. Yilmaz, International and Turkish information technology standardization, Computer Standards \& Interfaces 14 (1992) 401-409.

The International Organization for Standardization (ISO) is working towards establishing worldwide concensus-oriented standards in information technology. The report reviews the state-of-the-art in information technology standardization. It establishes that the current trend in both hardware and software is towards open platforms and open distributed systems. Networks are evolving towards intelligent networks. Financial institutions and network operating companies are cooperating in ISO committees to prepare security standards. In Turkiye the Turkish Standards Institute (TSE) is active more than ever in promoting the cause of standards. It is recognized that in the information technology area TSE's activities are limited to trying to have the product conformity to the Latin Alphabet No. 5 which includes Turkish characters at all levels. It is the wish of the industry leaders to introduce the present ISO information technology standards and contribute to the future standards. Various mechanisms are discussed on how this can be established.
\end{abstract}

Keywords: Information technology; base \& functional standards; character sets; bus standards; OSI; conformity certification; conformance testing; quality standards; ODA; ODP; Systems Application Architecture.

\section{Economic significance of global IT standard- ization}

In this report information technology is restricted to the most actively used fields of electronic documentation, databases, telecommunications, banking and accounting. As such information technology is dynamic of nature and evolves rapidly with technology. For example the reduction in cost of computer hardware over the years coupled with advances in distributed computing and computer network technologies have had a significant impact towards the decentralization and subsequent distribution of computer resources resulting in a distributed and open environment.

Rapid dramatic changes in technology increase the importance of standards. Standards provide

\footnotetext{
* Turkiye is used throughout the paper instead of Turkey Correspondence to: B. Sarikaya, Bilkent University, Dept. of Computer Engineering, and Information Sciences Bilkent, Ankara 06533, Turkey
}

the discipline necessary to ensure orderly evolution during technological improvement. At each stage of technology improvement, standards provide a stable platform for the next stage. This disciplined progress using standards dominates today's most important concept in information technology: the introduction and support of 'open systems'.

Standardization should be understood as voluntary, concensus-developed standards which are readily available to any vendor or user, are changed only under rules protecting the interests of all, and have no economic impediments to general use. Standardization process provides 'a level playing field' for all vendors and a stable environment for all users [28].

Standards are critically needed because they can reduce costs and allow users to choose from among a large selection of products. Without standards, confusion would result. For example, manufacturers of equipment would not have uniform physical or electrical performance parame- 
ters as guidelines to design their products to meet business needs. Users of nonstandard equipment would have to provide such features as physical communication interfaces, mountings, power requirements, and performance norms to accommodate each manufacturer's design.

The paper continues in Section 2 with a review of international IT standardization. Section 3 reviews IT standardization in a developing country, Turkiye. Section 4 offers the future plans and opinions on how developing countries can better introduce already-accepted IT standards and how they can participate in the preparation of the future standards. Finally Section 5 contains some concluding remarks.

\section{Trends in information technology standardiza- tion}

\subsection{Base and functional standards}

International Organization for Standardization (ISO) and Comite Consultatif International Telegraphique et Telephonique-Consultative Committee on International Telephone and Telegraph (CCITT) prepare base standards, the standards that contain the most general and common properties with all sorts of optional and mandatory features. Continental and national bodies prepare functional standards. Functional standards are certain profiles of base standards. They are prepared with implementation concerns and they represent certain choices of optional collection of features, i.e. profiles [20]. Functional standards must adhere to the relevant base standard, the mandatory conditional and optional functions must be properly designated and conformance to base standards must be supported. Here we should emphasize the importance of ISPs (International Standardized Profiles).

\subsection{Hardware related standardization}

In hardware, buses and interfaces are the two main places where standards have successfully been applied. The result has been to create open platform equipment for both users and vendors.

Buses and bus standards are playing an ever increasing role in the synthesis of computer based systems for a wide range of applications. The bus is a physical medium that interconnects processors, peripherals and memories. In this respect a bus is similar to a local/wide area network.

\subsection{Standards for quality assurance}

Quality systems are concerns of all industry including information technology industry. In more general context ISO has completed a series of standards (ISO 9000-9004) to define a model, guidelines and the rules for final inspection and quality assurance tests. Manufacturers of all sorts of goods are now required to follow these standards.

In the information technology area especially due to the abstractness of software, it is not clear how ISO 9000 series standards apply. Recently ISO JTC1 has directed its attention to this important subject and is in the process of preparing standards for software quality. ISO 9126 will be a standard on software product evaluation-Quality characteristics and guidelines for their use [5]. Software quality standard defines functionality, reliability, usability, efficiency, maintainability and portability as the six software quality characteristics. The importance of each quality characteristic varies depending on the class of software. For example, reliability is the most important for a mission critical software, efficiency is the most important for a time critical real time system software, and usability is the most important for an interactive end user software.

\subsection{Standards for software}

\subsubsection{Open Systems Interconnection}

Open Systems Interconnection (OSI) has dominated international standardization in telecommunications since 1976. OSI standardization has recently been completed with the standardization of the application layer protocols. Application layer is the layer 7 of the OSI Reference Model. Key application layer standards are message handling systems (MHS), file transfer and access management (FTAM), the directory and transaction processing (TP).

\subsubsection{Open software in workstation environment}

The dominant operating system in workstation environment is Unix ${ }^{1}$. There are several window-

\footnotetext{
${ }^{1}$ Unix is a trademark of AT\&T.
} 
ing systems that run under Unix. Present trend is a convergence towards the $\mathrm{X}$ Window System, Version 11.

Open Software Foundation and IEEE are working towards bringing global acceptance to a single standard for open software. OSF/Motif and IEEE Portable Operating System Interface for Computer Environment (Posix) are the current results of these efforts. While OSF/Motif has wider range scope, IEEE Posix is scoped to Unix environment.

POSIX efforts have so far produced an international standard on an application programming interface in the C language [19]. ISO 9945 represents the language approach to the definition of APIs. ODP which is discussed next represents the conceptual approach.

\subsubsection{Open distributed processing}

Traditional telephone networks are evolving to become intelligent networks. While present telephone networks are oriented towards serving basic telephone calls, the future networks will be oriented towards services such as 'freephone' which will allow reverse charging, "virtual private network' which will permit to build a private network by using public network resources, and 'universal personal telecommunications' which will enable subscribers to make use of telecommunication services on the basis of a unique personal telecommunication number which will be network independent. The key objectives of universal personal telecommunications (UPT) are to obtain network programmability and to provide some openness in service and equipment provision. This is obtained by dissociating the control part of the network in a stand-alone subnetwork and simultaneously providing this subnetwork with standardized means of control on the switching resources. The advantages are network-wide control, programmability, smooth integration of existing equipment. UPTs various processing entities can be conceptualized in terms of the logical functions of call-control, service-control, and management-related functions [25]. Call control comprises CCF (Connection Control Function) and SSF (Service Switching Function) and SSF recognizes special calls to route them to SCF (Service Control Function). Management related functions include SMF (Service Management Function) which is the counter part of SCF for the management part of services and SCEF (Service Creation Environment Function) modelling the ability to create new services [2].

UPT requires the development of new services independently of network equipment vendors and and equipments. Open distributed processing (ODP) standardization is in progress in $\mathrm{SC} 21$ of ISO in order to define conceptual framework in which UPT requirements can be met [23].

\subsubsection{Coding related standards}

A character is a member of a set of elements used for the organization, control and representation of data. Characters are grouped into character sets. There must be one-to-one relationship between the characters of the set and the codes assigned to them. Fixed length (7-, 8-, and 16-bit long) coding is used most. ISO 646 is a 7-bit coding standard which is commonly known as ASCII. ASCII incorporates English language but it does not handle other Latin alphabets. ISO 8859 is a series of standards that uses 8-bit coding and incorporates several Latin alphabets. Latin Alphabet No. 1 is part 1 of this standard. Latin 1 is the coding that is supported by all the manufacturers doing business in Europe. The coding of Latin Alphabet No. 1 is accepted in several other contexts also. ISO 7810 series standards on identification and credit cards incorporate or recog. nize the need for Latin 1 encoding [4].

16-bit character encoding standards are also being prepared. ISO TC68/SC2 is preparing a universal 16-bit code set which will be known as ISO 10646.

\subsubsection{ODA standards}

Open Document Architecture (ODA) is an international standard for integrating office systems [10]. ODA is an interchange standard for multimedia documents which has been produced in order to allow such documents to be exchanged between conforming computer systems anywhere in the world. Using the ODA standard, electronic documents can be used and transferred worldwide, being imaged (e.g. printed or displayed) or processed (e.g. edited or reformatted) by the recipient according to the intentions of the originator [3].

The format for storage and transfer of documents is known as Office Document Interchange Format (ODIF). Systems can be based on the 
direct use of ODA architecture or can use their own information architecture and convert to and from ODIF for archive storage or transfer of documents. ODA defines a precise model to view a document structure: The logical structure which relates the content of a document to constituents such as paragraphs, headings and footnotes and the layout structure which relates content to its layout characteristics, such as type fonts and pagination. Logical and layout structures are hierarchies of objects, each object being described by a set of attributes.

\subsubsection{Security related standards}

The move towards distributed and open environments provides an appealing target for intruders wishing to gain access to or modify sensitive information to their advantage. This brings interest in the area of network security [24]. ISO's 7498-2 OSI security architecture defines the general security related architectural elements that can be applied appropriately in the circumstances for which protection of communication is required. It provides the mechanisms of authentication, access control, data confidentiality, data integrity and nonrepudiation (repudiation is the denial by one of the entities involved in a communication of having participated in all or part of the communication). Main security techniques are enchipherment (encription) and digital signature.

\subsubsection{Conformance testing}

Conformance testing of OSI protocol implementations has been an active area for standardization since 1983. First a standard on the methodology and framework of conformance testing has been developed [18]. Conformance test suites written in the conformance test specification language TTCN [26] for OSI protocols are also standardized themselves. TTCN is Part 3 of [18]. Conformance test suite standards for each layer of OSI are being developed. Session layer standard contains 4 parts [22]. Conformance testing has brought the need for defining a Protocol Implementation Conformance Statement (PICS) proforma for each and every protocol standard. PICS proformas are being added to the protocol standards, for example Part 4 is the PICS proforma of the distributed transaction processing protocol standard [21].

\subsection{Standardization in Europe, the US and Japan}

Traditionally Europe was always more motivated towards standardization than any other part of the world because of the existence of high number of countries with very close economic and social ties. Business complained about differing regulations and standards in each and every country. So, as a result, Europe has always led standardization activities. This is especially the case in OSI standardization. Presently Europe, the US and Japan are all cooperating in ISO and CCITT since European acceptance of these standards will mean the introduction of conforming products in European market and that it is to everybody's advantage to standardize.

Apart from participating in ISO and CCITT, Europe has its own organizations for standardization. CEN (Committee for European Normalization) and ETSI (European Telecommunications Standards Institute) are among the most important. The primary task of ETSI is to define uniform telecommunications standards for Europe, to be adopted by each member state, in order to link up national networks and services and thereby establish a pan-European telecommunications infrastructure to benefit both the user and manufacturing communities [27]. ETSI work does not try to reinvent the wheel. Where necessary, it builds upon the best standards already in existence elsewhere (ISO and CCITT) and tailors them to suit common European requirements. Therefore ETSI builds upon base standards to prepare Europe-wide functional standards. Today's work program of ETSI includes about 200 standards to enable commercial operation of the Integrated Services Digital Network (ISDN), about 100 standards for Digital Cellular Network, and others on broadband, satellite communications and intelligent networks [6].

\section{IT standardization activities in Turkiye}

The Turkish Standards Institute (Turkiye Standardlar Enstitusu) TSE is a participating (P-) member to several of ISO/IEC JTC1 Special Committees (SC) including SC17 on identification and credit cards. Recently it has changed its status from observer $(\mathrm{O})$ to $\mathrm{P}$-member in one of the most active SCs, SC21. TSE is organizing the 
next annual meeting of SC17 in Istanbul in October 1992.

TSE recognizes the fact that it should do more in IT standardization. It is also prepared to participate more actively on the discussions regarding Turkish participation in this field. These points will be elaborated in Section 4 below.

ISO 9000 series standards on quality assurance have been adopted by TSE. The TSE has given a different number to the resulting standards-TS 6000 series standards. These standards are among the most popular in Turkish industry. Several manufacturing companies have acquired these standards. As far as the conformity to the quality assurance standards, TSE has so far given certificates to only two institutions.

TSE gives special attention to character set standards because Turkish alphabet has distinct characteristics than any other Latin alphabets. Unfortunately Latin Alphabet No. 1 (ISO 8859/1) does not fully cover the Turkish Alphabet. Recently Latin Alphabet No. 5 (ISO 8859/5) has been defined which covers fully the Turkish alphabet. Latin 5 has been standardized as TSE 5581 [30]. Latin 5 code is obtained by modifying Latin 1 code table in 6 places to accommodate $\breve{\mathrm{G}}$, ğ (capital and small-case mild-g), Ş, ş (capital and small-case character pronounced 'sh'), I (capital vowel pronounced ' $\mathrm{e}$ '), and i (small-case vowel I pronounced as in Iraq). The resulting code table is shown in Appendix A. Latin 5 contains complete character sets of a total of 44 countries including Europe excluding east Europe. Latin 1 and Latin 5 are presently competing standards. There seems to be no reason for their coexistence. TSE expects that future developments will bring stronger international acceptance for Latin 5 and that Latin 5 will supersede Latin 1.

Turkiye has a number of strong banks and financial institutions. These institutions are integrated with their international counterparts. Identification and credit card standards therefore need to be commonly recognized by these institutions. Recently, with the leadership of the Banking Association which brings together all the banks and financial institutions, TSE is adopting ISO 7816 series standards on identification and credit cards. The newer standards for integrated circuit cards will also be adopted. In adopting these standards TSE makes sure to incorporate Latin 5 wherever needed [29].
Another important development led by the Banking Association has been the establishment of the Electronic Funds Transfer (EFT) which provides interworking of information exchange among banks and financial institutions. EFT has become a reality with an international effort that provided both equipment and software to achieve this interworking through international standards.

TSE is presently not much involved with telecommunications standardization. Telecommunications is one of the key industries of the country. The telecommunications industry is presently involved in manufacturing of all sorts of equipment. Software production for telecommunications is just starting. Some of the companies have adopted CCITT's formal specification language SDL defined in Z.100 series standards and making progress towards integrated telecommunications software engineering based on SDL [31]. All of the telecommunications companies recognize the importance of standardization at the global level and in specific for the European market. The companies are participating in ETSI activities. This participation is at times productive. Turkish contributions are known to have improved some of the ETSI standards [1].

ETSI standards are widely accepted by the country's telecommunications industry. But at present no ETSI standards that been translated into Turkish and no standard has gone through national modification.

\section{Future of IT standardization in Turkiye}

This section is an extract of ideas collected from interviews conducted by the authors with the industry leaders. While the reference is made to a specific country, it is believed that most of the ideas apply to developing countries in general.

\subsection{Common industry concerns}

Common concerns of all industries are in the establishment of software fault tolerance. This common concern is probably due to a traditionally high rate of power failures and infrastructural deficiencies in Turkiye as well as in other developing countries. 
It is the wish of the IT industry that TSE play more active role in introducing current IT standardization activities to the industry.

Personal computer (PC) software companies are concerned with Latin Alphabet No. 5. The desire here is to see more hardware support by PC manufacturers, otherwise supporting Turkish alphabet by software increases software development time and decreases the competitiveness of the companies.

Integrated Accounting and Taxing is an area where local companies are more developed possibly due to the national laws and regulations in this area. Local companies have a higher market share in this area. Their products are well developed. These companies would like to follow closely the IT standardization since they see Europe as possible future market for their products.

The main concern of industry in integrated accounting and taxing is the domain specific standards deficit in business data processing. There are more than one accepted proformas of main business accounting such as grand account-books, inventory and balance sheet. The companies doing business in this sector are required to support all these proformas and this increases software development time and obviously reflects to the cost.

As far as information technology expertise is concerned, the industry is relying mostly on new graduates from Universities. These newly graduated software engineers are highly deficient in documentation preparation. It is the concern of industry that qualified personnel to prepare users and programmers manuals for their products is difficult to find.

Banking industry is interested in workstation environment and plans to shift the information processing to workstations. In this respect IEEE/Posix and OSF/Motif are important steps to follow.

\subsection{Trade barriers for software houses}

Presently the IT market in the country is dominated by import companies and this is not expected to change in the short term. The government requires no excise tax for software but the value added tax applies to all institutions except those that are exempt. Government requires bidding for all acquisitions higher than $\$ 50,000$.
Commonly used PC software is marketed by the originating companies. The price for such software is approximately $30 \%$ higher than international prices. Given the lower income level of the customers, these prices look even higher than in developed countries.

Another concern here is the copyright rules for software. At the judicial level the Turkish laws are modern and they recognize the software copyright. In practice, it is recognized that the laws must be reinforced more rigorously and that a public awareness must be established. Industry associations are putting forward several steps in this regard. Television and newspaper campaigns are expected to increase the attention of the public. It will possibly take time until complete awareness to software copyright is established. For the industry, it becomes important to provide the products cheaper and also take measures to avoid copyright violations.

\subsection{Recommendations}

We suggest several actions which will promote standardization. Turkish government should adopt the rule of buying information technology standards conforming products just as the US government does. This move is expected to bring the boost required to bring the necessary attention to information technology standardization country-wide.

Office Document Architecture can be imposed as the standard way of storing and interchanging office documents to be provided to the government. There is a growing need to do this especially in the area of periodic (monthly) information transfers from all sorts of companies to the government for social security.

Tax incentives can be established for exportations of software produced in the country. This will provide first of all parity with all other sectors of manufacturing and will encourage the establishment of a healthy software industry.

Present attention of the IT industry and TSE for standardization is concentrated on coding the Turkish alphabet. Once Latin Alphabet 5 is more recognized internationally, IT industry should direct its attention to more technical issues. This will become possible only with the cooperation of experts from Universities, TSE and industry. 
Annual conferences on computer science are expected to play an important role in the process of the introduction of IT standards to the industry and to the users. These conferences may as well serve in more active participation in IT standards preparation. Encouragement of the contributing individuals through awards programs can also be achieved in these annual events.

Industry associations are also mentioned as possible places where IT standards can be introduced and awareness to these standards and present international trends can be established. For more active participation in the preparation of IT standards the importance of national committees to study each effort from the very beginning can not go unrecognized. Industry associations can lead the establishment of such committees. When the understanding and contribution reaches to a certain level, the committee can send representative(s) to the corresponding ISO/CCITT or ETSI committee.

For Turkiye it is recognized that there are certain sectors of IT that look at present more important than others. These sectors are telecommunications, banking, medical software, tourism and accounting. There seems to be sufficient accumulation of expertise in these sectors to both introduce and then to contribute to the IT standardization.

Finally standardization and quality assurance naturally bring to the scene the importance of conformity of the products marketed to the IT standards. In telecommunications, conformance testing of the implementations can be done by conformance test centers. Europe is leading in conformance testing and is establishing several test centers [27]. In Turkiye the importance of conformance testing should be recognized and both government and telecommunications industry should adopt the rule of buying conformance tested/certified products. Telecommunications industry should play active role in the preparation of conformance test standards.

TSE is presently issuing certificates to the companies that conform to certain standards. This activity should be extended to IT sector. Like its counterparts in industrialized countries TSE should play active role in the development of test suites and procedures for all IT standards and should start the process of IT certification.
Here the two areas should be distinguished: telecommunications and other IT standards. Telecommunications sector conformity certification requires active testing thus more involved investment. This can only be done with cooperation with industry. Other IT sectors require passive conformity procedures. TSE should lead the development of the conformity procedures and/ or should adopt existing international ones and should base its certification program on these procedures.

\section{Concluding remarks}

The goals for IT standardization are to eliminate the costs to Turkish businesses of incompatibilities and/or the constraints on systems which derive from incompatibilities, to provide common functionality across systems, to have an assured functionality, to make it possible to use IT equipment from different sources in combination, to form a technical basis for developing procurement specifications, to promote an open market within Turkiye in equipment and services, reducing the scope of protectionism and to contribute in the reduction in the costs of development and maintenance of equipment and systems.

Introduction and promotion of IT standards in individual countries require functional standards to be accepted by National Bodies. The Turkish National Body, TSE, has concentrated its efforts on the character set standard that incorporates Turkish characters, i.e. the Latin Alphabet No. 5. In telecommunications area, regional functional standards prepared by ETSI have wide spread acceptance in telecommunications industry. Industry associations are expected to play an active role in the process of introducing and promoting IT standards. As this progresses TSE will start producing national functional standards.

In the process of standards preparation developing countries are expected to concentrate on the areas of national importance. As the reduction in the costs of IT equipment and services is materialized, the information technology will be more accessible to the developing countries. This in turn is expected to increase participation in the preparation of new IT standards. 


\section{References}

[1] A.R. Akcay, Some proposals for new transport technology in BISDN, in: E. Arikan, (ed) Communication, Control, and Signal Processing (Elsevier Science, Amsterdam, 1990) $1-7$.

[2] G. Bregant, Towards a convergence between telecommunication services and open distributed processing, J.d. Meer and V. Heymer, eds., Internat. IFIP Workshop on $O D P$, Berlin (Oct. 1991).

[3] I.R. Campbell-Grant, Introducing ODA, Comput. Standards \& Interfaces 11 (3) (1991) 149-157.

[4] M. d'Cruz, E. Kulinek and E. Lee, Character sets of today and tomorrow, Comput. Standards \& Interfaces, 8 (3) (1989) 199-208.

[5] Standardization activities, Comput. Standards \& Interfaces 11 (3) (1991) 215-255.

[6] European Telecommunications Standards Institute (ETSI), ETSI Work Program (1991).

[7] L. Guzenda and A.E. Wade, A taxonomy of standards, Comput. Standards \& Interfaces 13 (1-3) (1991) 65-70.

[8] ISO, ISO 7-bit Coded Character Set for Information Exchange (1983).

[9] ISO/IEC/JTC 1, Identification Cards-IC Cards with Contacts, Parts 1-3 (1991).

[10] ISO, Office Document Architecture (ODA) and Interchange Format, Parts 1-8 (1991).

[11] ISO, 8-bit single Byte Coded Graphic Character Sets-Part 1: Latin Alphabet No. 1 (1986).

[12] ISO, 8-bit Single Byte Coded Graphic Character Sets-Part 1: Latin Alphabet No. 5 (1988).

[13] International Standards Organization (ISO), Quality Management and Quality Assurance Standards; Guidelines for Selection and Use (1987).

[14] International Standards Organization (ISO), Quality Systems; Model for Quality Assurance in Design / Development, Production, Installation and Servicing (1987).

[15] International Standards Organization (ISO), Quality Systems; Model for Quality Assurance in Production and Installation (1987).

[16] International Standards Organization (ISO), Quality Systems; Model for Quality Assurance in Final Inspection and Test (1987).

[17] International Standards Organization (ISO), Quality Management and Quality System Elements; Guidelines (1987).

[18] ISO/IEC/JTC1, Conformance Testing Methodology and Framework, Parts 1-7 (1992).

[19] ISO/IEC JTC1, Portable Operating System Interface (POSIX) Part I: System Application Program Interface (API) [C Language] (1990).

[20] ISO/IEC TR, Framework and Taxanomy of Internation Standardized Profiles, Parts 1-2, (1991).

[21] ISO/IEC/JTC1, Distributed Transaction Processing, Parts 1-4 (1992).

[22] ISO/IEC/JTC1, Conformance Test Suite for the Session Protocol, Parts 1-4 (1992).

[23] ISO/IEC/JTC1, Basic Reference Model of Open Distributed Processing, Parts 1-5 (1992).
[24] A. Patel, Emerging network security standards in an OSI environment, Comput. Standards \& Interfaces 9 (3) (1990) 239-247.

[25] Proposed Draft CCITT Q.1200 Intelligent network recommendation, June 1991, Geneva.

[26] B. Sarikaya and A. Wiles, Standard conformance test specification language TTCN, Comput. Standards \& Interfaces 14 (2) (April 1992) 117-144.

[27] The SPAG Standard, European Newspaper for OSI Conformance Testing Activities, Autumn 1991 issue.

[28] E.L. Stull and J.L. Berg, The role of standards, Comput. Standards \& Interfaces 13 (1-3) (1991) 9-16.

[29] Turkish Standards Institute, Catalogue of Turkish Standards TSE'91 (1991) (in Turkish).

[30] Turkish Standards Institute, 8-bit Single Byte Coded Graphic Character Set (1988) 4 pages (in Turkish).

[31] CCITT, Specification and Description Language, Z.100 (1992).

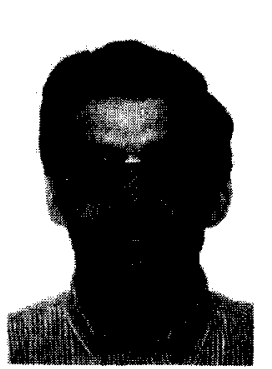

Behçet Sarikaya received his B.S.E.E. degree (honors) from the Middle East Technical University (METU) Ankara. Turkey in 1973, M.Sc. degree in Computer Science from METU in 1976, and Ph.D. degree in Computer Science from McGill University, Montreal, Canada, in 1984.

He worked in the Universities of Sherbrooke and Concordia as Assistant Professor. He is presently working in the Department of Computer and Information Sciences, Bilkent University, Ankara. He has published numerous papers in protocol engineering and related areas. He has been co-chairman of the conference IFIP PSTV VI held in Montreal in 1986. He served in the program committees of all three protocol conferences. His current research interests lie in all aspects of conformance testing and high-speed networks. $\mathrm{He}$ is a senior member of IEEE.

$\mathrm{He}$ is actively involved in OSI standardization activities. $\mathrm{He}$ is an active member of the joint CCITT SG X Question 10, ISO IEC JTCI/SC21 Project 54 on Formal Methods in Conformance Testing. This committee aims at developing a standard that will relate the Formal Description Techniques to the Conformance Testing Methodology and Framework.

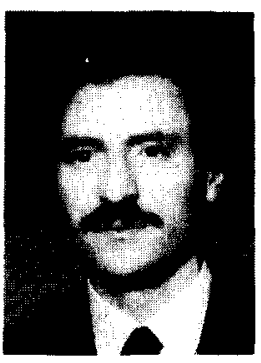

Rasim Yilmaz received his B.S.E.E. degree from Istanbul State Eng. and Arch. Academy (Yildiz University) Istanbul, Turkey in 1980, MSc degree in Information Science from Loughborough University of Technology, UK in 1989. His job experience includes Afşin-Elbistan Thermal Power Plant as field engineer between 1981 1984, Turkish Elektromechanical Industries Corp. as design engineer between 1984-1985, Modern Company, Riyad Saudi Arabia as field engineer between 1985-1986, Turkish Electronic Industries Corp. as chief electrical engineer between 1986-1987. Presently he is in charge of Computer and Information Dept. Turkish Standard Institution (TSE) since 1989. 


\begin{tabular}{|c|c|c|c|c|c|c|c|c|c|c|c|c|c|c|c|c|}
\hline & & & $\frac{10}{\frac{1}{0}}$ & $\frac{1}{27}$ & 0 & $\frac{0}{1}$ & $\frac{1}{\frac{1}{0}}$ & & \begin{tabular}{|l|l|}
0 \\
0 \\
0 \\
\end{tabular} & $\begin{array}{l}10 \\
0 \\
0 \\
\end{array}$ & \begin{tabular}{|c|}
0 \\
$\frac{1}{0}$ \\
0
\end{tabular} & $\frac{1}{1}$ & $\frac{1}{1}$ & $\frac{1}{1}$ & $\frac{1}{1}$ & $\begin{array}{r}1 \\
\frac{1}{1} \\
\frac{1}{1} \\
\end{array}$ \\
\hline 951 & & 0001 & 02 & 33 & 04 & 05 & 06 & 07 & 108 & 09 & 10 & 11 & 12 & 13 & 14 & 15 \\
\hline 000 & 00 & & SP & 0 & a & $P$ & & $p$ & & & NASP & • & $\dot{A}$ & $\Xi$ & à & $\breve{s}$ \\
\hline 0001 & 01 & & $!$ & 1 & $\mathrm{~A}$ & $Q$ & $a$ & 9 & & & $i$ & \pm & Á & \begin{tabular}{|l|}
$\tilde{\mathbf{N}}$ \\
\end{tabular} & $\dot{a}$ & $\tilde{n}$ \\
\hline 0 & 02 & & $"$ & 2 & B & $R$ & $b$ & $r$ & & & 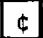 & 2 & $\hat{A}$ & ì & â & $\dot{0}$ \\
\hline \begin{tabular}{l|l|}
0 & 1 \\
\end{tabular} & 03 & & $\not$ & 3 & C & $\mathrm{s}$ & c & $s$ & & & $E$ & 3 & $\bar{A}$ & $\dot{0}$ & \begin{tabular}{|l|}
$\tilde{a}$ \\
\end{tabular} & ó \\
\hline 0,100 & 04 & & $\$$ & 4 & 0 & $T$ & d & $t$ & & & $\square$ & 1 & A' & $\hat{0}$ & ä & 0 \\
\hline 101 & 05 & & $\%$ & 5 & $E$ & $U$ & $\mathrm{e}$ & $u$ & & & 7 & $\mu$ & 8 & $\tilde{0}$ & a & $\tilde{0}$ \\
\hline$\sqrt{1}$ & 06 & & $\&$ & 6 & $F$ & $v$ & $f$ & $v$ & & & $i$ & 4 & $F$ & 0 & $\mathrm{a}$ & 0 \\
\hline 0 & 07 & & 1 & 7 & $G$ & $w$ & $\mathrm{~g}$ & $w$ & & & 5 & - & 5 & $x$ & $c$ & $\div$ \\
\hline 000 & 08 & & 6 & 8 & $H$ & $x$ & $h$ & $x$ & & & $"$ & & $\bar{E}$ & 0 & è & $\theta$ \\
\hline 100 & 09 & & 2 & 9 & $I$ & $Y$ & $i$ & $y$ & & & (c) & & $E$ & ن & é & $\dot{U}$ \\
\hline 101 & 10 & & $\star$ & $\therefore$ & 1 & 2 & $j$ & 2 & & & $!$ & 9 & $\hat{E}$ & $\dot{U}$ & ê & Ú \\
\hline $1[0] 11$ & 11 & & + & ; & $k$ & [ ] & $k$ & 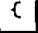 & & & $\ll$ & $\gg$ & $\ddot{E}$ & 0 & ë & $\hat{u}$ \\
\hline 100 & 12 & & 1 & $<$ & L & 1 & l & 1 & & & 7 & $1 / 4$ & ì & uj & $i$ & $\ddot{u}$ \\
\hline 101 & 13 & & - & $=$ & $M$ & 3 & $\mathrm{~m}$ & 3 & & & sin & $1 / 2$ & I & I & $i$ & 1 \\
\hline+ & 14 & & $\cdot$ & 3 & $N$ & - & $n$ & - & & & (1) & $3 / 4$ & I & 5 & $\hat{i}$ & 5 \\
\hline 11 & 15 & & 1 & $?$ & 0 & & $\circ$ & & & & - & $i$ & $i$ & B & & $\ddot{y}$ \\
\hline
\end{tabular}

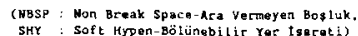

